\title{
Electroweak Precision Measurements with the ATLAS Detector
}

\section{Zhiqing Zhang ${ }^{* \dagger}$}

LAL, Univ. Paris-Sud, CNRS/IN2P3, Université Paris-Saclay, Orsay, France

E-mail: zhangzq@lal.in2p3.fr

\begin{abstract}
With the high integrated luminosities recorded at the LHC and the very good understanding of the ATLAS detector, it is possible to measure electroweak observables to the highest precision. In this talk, we present the tau polarisation, measured in $Z \rightarrow \tau \tau$ using $20.3 \mathrm{fb}^{-1}$ of proton proton collision data collected at a centre of mass energy of $8 \mathrm{TeV}$. The talk also reviews the measurement of forward-background asymmetry based on the triple differential Drell-Yan cross-section obtained with the same data sample, which can be used to extract the weak mixing angle. We conclude with a presentation of the measurement of the $W$-boson mass using $4.6 \mathrm{fb}^{-1}$ data, collected at $7 \mathrm{TeV}$.
\end{abstract}

XXVI International Workshop on Deep-Inelastic Scattering and Related Subjects (DIS2018) 16-20 April 2018

Kobe, Japan

\footnotetext{
* Speaker.

${ }^{\dagger}$ on behalf of the ATLAS Collaboration.
} 


\section{Introduction}

The Standard Model (SM) has a number of free parameters. In addition to the Higgs boson mass $\left(m_{H}\right)$, the fermion masses and mixings, and the strong coupling constant $\left(\alpha_{s}\right)$, the set of three parameters with the smallest experimental errors contains the fine structure constant $(\alpha)$, the Fermi constant $\left(G_{\mathrm{F}}\right)$ and the $Z$-boson mass $\left(m_{Z}\right)$. The values of other electroweak (EW) parameters, e.g. the weak mixing angle $\left(\sin \theta_{W}\right)$ and the $W$-boson mass $\left(m_{W}\right)$, may be derived from these three parameters as $\sin ^{2} \theta_{W}=1-m_{W}^{2} / m_{Z}^{2}$ in the on-shell scheme and $m_{W} \sin ^{2} \theta_{W}=\pi \alpha /\left[\sqrt{2} G_{\mathrm{F}}(1-\Delta r)\right]$, where $\Delta r$ includes high-order radiative corrections. These EW parameters can also be directly determined through observables that depend on them, e.g. $m_{W}$, forward-backward asymmetry $\left(A_{\mathrm{FB}}\right)$ and lepton asymmetry parameter $\left(A_{\ell}\right)$. A comparison between the indirect and direct determinations allows a non-trivial test of the SM. The precision measurements are sensitive to new physics independent of direct searches. So far the indirect determination of all these observables is still more precise than that of the measurement and also the $A_{\ell}$ measurements from LEP and SLC have an important discrepancy [1]. This motivates the experimental efforts by ATLAS [2] at the LHC in measuring the tau polarisation $P_{\tau}$ in $Z$-boson decays [3] (related to $A_{\tau}$ ), the $A_{\mathrm{FB}}$ through triple-differential cross section of Drell-Yan events [4] and the $W$-boson mass [5] described in the following.

\section{Measurement of tau polarisation}

The tau $(\tau)$ lepton is the heaviest lepton in the SM. Its polarisation, defined as the asymmetry of the cross section for positive and negative helicity $\tau$ production, is a measure of the degree of parity violation in the interaction producing the $\tau$ leptons. It provides therefore insight into the nature of its Lorentz structure. It is proportional to $1-4 \sin ^{2} \theta_{\text {eff }}$ where $\sin ^{2} \theta_{\text {eff }}=\kappa \sin ^{2} \theta_{W}$ with $\kappa$ accounting for higher-order corrections. The measurement is performed with $\tau$ leptons produced in $Z / \gamma^{*} \rightarrow \tau \tau$ decays based on a dataset of $p p$ collision at a centre-of-mass energy $(\sqrt{s})$ of $8 \mathrm{TeV}$, corresponding to an integrated luminosity of $20.2 \mathrm{fb}^{-1}$. The leptonic decays of one of the taus are used as tag and the one-prong hadronic decay modes of the other tau are used as the polarisation analyser defining $\Upsilon=\left(E_{\mathrm{T}}^{\pi^{ \pm}}-E_{\mathrm{T}}^{h^{0}}\right) / E_{\mathrm{T}}^{\tau_{\text {had }- \text { vis }}}=2 p_{\mathrm{T}}^{\text {track }} / E_{\mathrm{T}}^{\tau_{\text {had-vis }}}-1$ as the experimental observable. Here $h^{0}$ stands for neutral hadrons, $p_{\mathrm{T}}^{\text {track }}$ and $E_{\mathrm{T}}^{\tau_{\text {had-vis }}}$ are the transverse momentum of the one-prong track and the transverse energy of the visible hadronic energy, respectively. The distribution of the muontagged sample is shown in figure 1a. The asymmetry between the left- and right-handed helicities (dominated by the $h^{ \pm} \pi^{0}$ mode) is clearly visible. The dominant background from $W+$ jets and multijets is estimated using data-driven methods with dedicated control samples, whereas the minor background is estimated from simulation. The Likelihood profiles of the polarisation for the electron and muon-tagged channels and their combination in the selected mass range of $66-116 \mathrm{GeV}$ are shown in figure $1 b$. The combined polarisation value of $-0.14 \pm 0.02$ (stat) \pm 0.04 (syst) agrees with SM prediction of $-0.1517 \pm 0.0019$ based on the ALPGEN event generator interfaced with PYTHIA6 parton shower modelling and the TAUOLA $\tau$ decay library [3].

\section{Triple-differential Drell-Yan cross sections and forward-backward asymmetry}

Using the same $8 \mathrm{TeV}$ dataset as above, a measurement of the triple-differential cross section 

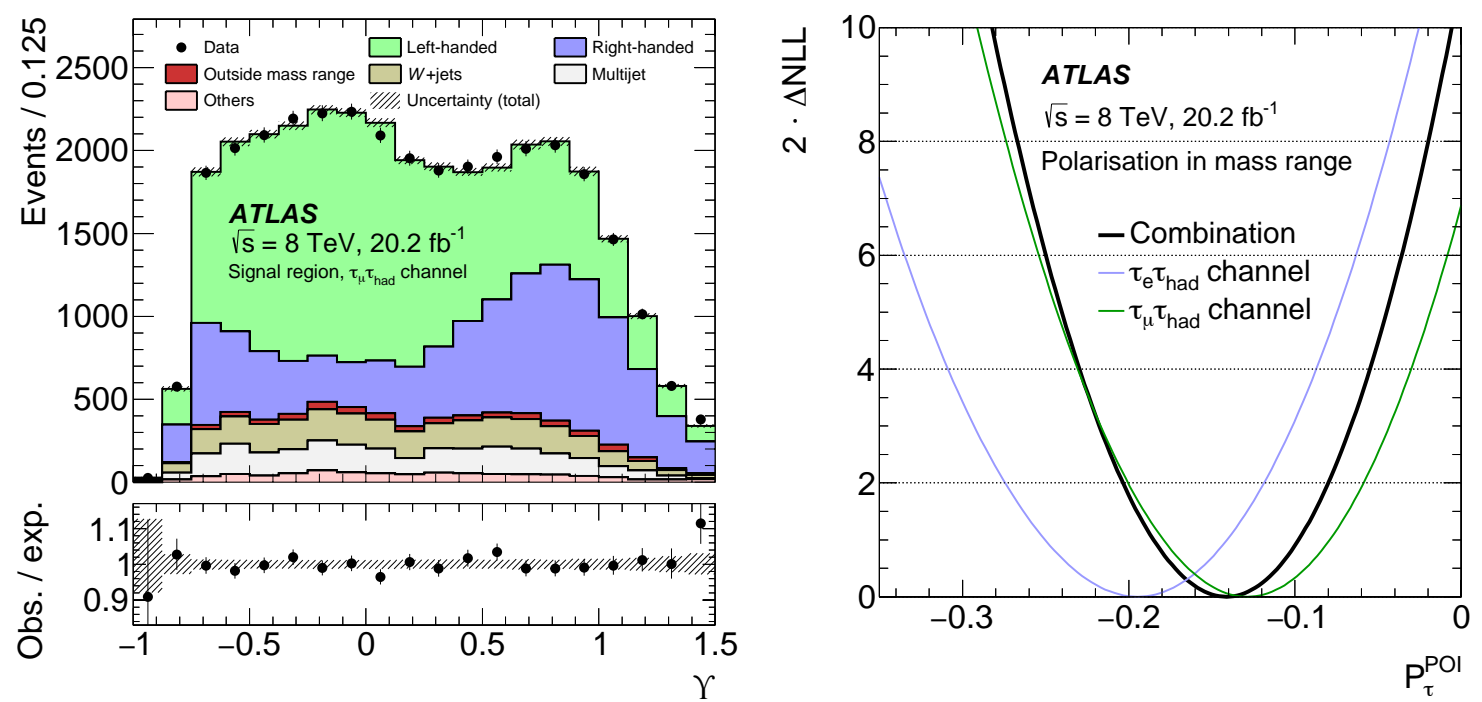

Figure 1: Left: Post-fit $\Upsilon$ distribution for $\tau_{\mu} \tau_{\text {had }}$ channel. Right: likelihood profiles of $P_{\tau}^{\text {POI }}$ for the fits that extract the polarisation in the mass-selected region of $66<m_{Z / \gamma^{*}}<116 \mathrm{GeV}$. Both plots are taken from [3].
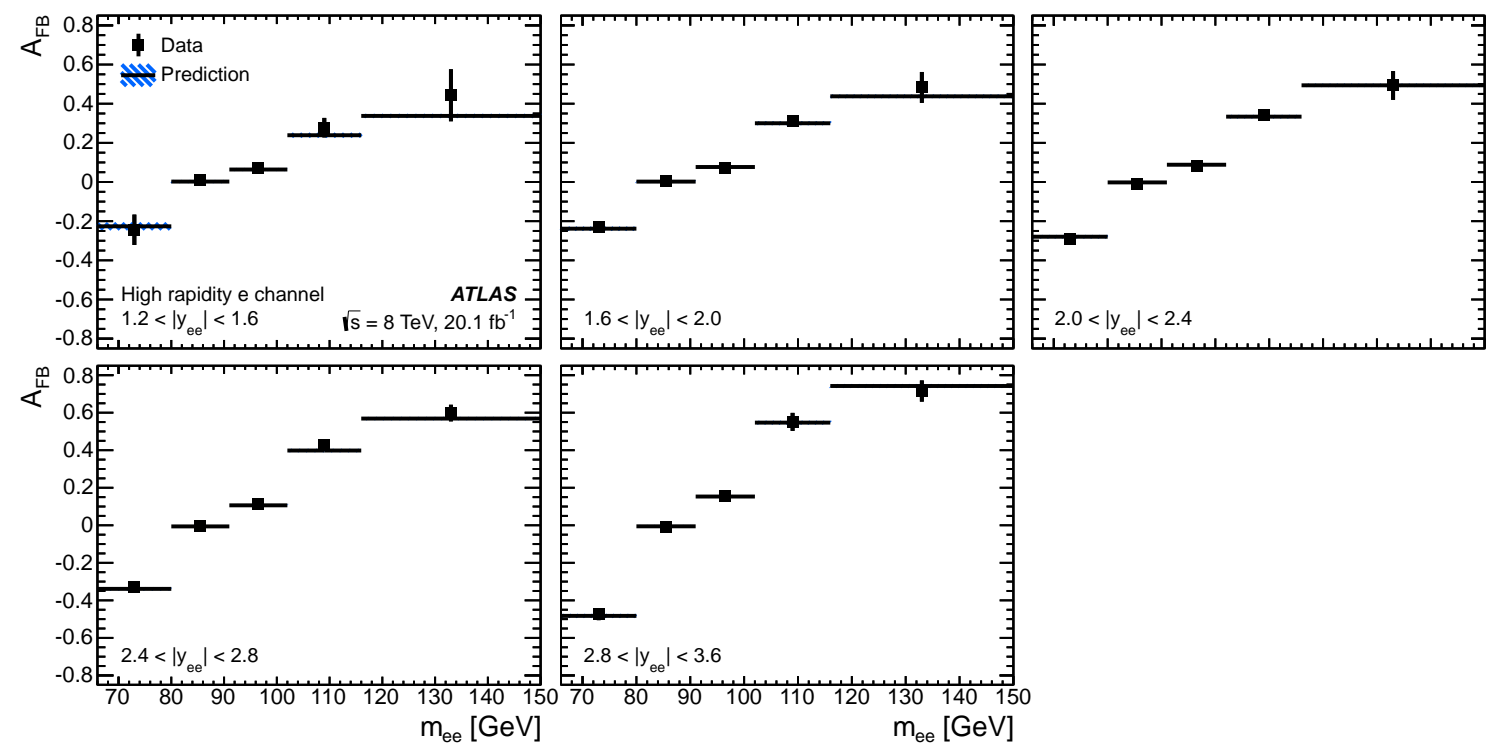

Figure 2: Forward-background asymmetry $\left(A_{\mathrm{FB}}\right)$ determined from the high-rapidity electron Born-level fiducial cross section [4]. The data are shown as dots and the error bars represent the total experimental uncertainty. The prediction from POWHEG including NNLO QCD and NLO EW corrections is shown as the solid line and the hatched band represents the statistical and PDF uncertainties. 
for the Drell-Yan process $Z / \gamma^{*} \rightarrow \ell^{+} \ell^{-}$, where $\ell$ is an electron or a muon, is performed in terms of invariant masses of the lepton pairs $\left(m_{\ell \ell}\right)$ in 7 bins between 46 and $200 \mathrm{GeV}$, absolute dilepton rapidity $\left(\left|y_{\ell \ell}\right|\right)$ in 12 bins between 0 and 2.4 , and the angular variable $\cos \theta^{*}$ between the outgoing lepton and the incoming quark in the Collins-Soper frame in 6 bins between -1 and 1 . The advantage of the Collins-Soper frame is that this choice of an angle is less sensitive to initial state radiation of the incoming partons. Due to the radiation, the quark direction may no longer align with the initial proton direction. The cross sections are used to determine the $Z$ boson $A_{\mathrm{FB}}$ as a function of $m_{\ell \ell}$ and $\eta_{\ell \ell}$ extending up to 3.6 in the electron channel (figure 2). The cross section measurements achieve high-precision, below the percent level in the pole region, excluding the uncertainty of $1.9 \%$ in the integrated luminosity. The $A_{\mathrm{FB}}$ measurements have even higher precision due to the cancellation of some of the systematic uncertainties and are dominated by statistical uncertainty. These precision data are sensitive to the parton distribution functions (PDF) and the effective weak mixing angle. In particular the $A_{\mathrm{FB}}$ measurements at low and high $m_{\ell \ell}$ are more sensitive to PDFs while the measurements at intermediate mass around the $Z$ resonance peak are more sensitive to the mixing angle [6].

\section{Measurement of $W$-boson mass}

A measurement of the $W$-boson mass using the $p p$ collision data collected by ATLAS at $7 \mathrm{TeV}$ corresponding to $4.6 \mathrm{fb}^{-1}$ is obtained through template fits to the reconstructed distributions of the charged lepton transverse momentum $\left(p_{\mathrm{T}}^{\ell}\right)$ and of the $W$ boson transverse mass $\left(m_{\mathrm{T}}\right)$ in the electron and muon decay channels. The measurement relies on a thorough detector calibration based on the study of Z-boson events, leading to a precise modelling of the detector response to electrons, muons and the hadronic recoil. Templates for the $W$-boson kinematic distributions are obtained from the NLO MC generator Powheg, interfaced to Pythia8 for the parton shower. The signal samples are supplemented with several additional physics-modelling corrections allowing for the inclusion of higher-order QCD and EW corrections. Two kinematic distributions used in the fits are shown in figure $3 \mathrm{a}$ and $\mathrm{b}$. The $W$-boson mass is obtained in a large number categories corresponding to different flavours, $W$-boson charges, kinematic distributions and rapidity regions. The consistency of the results in the electron and muon channels provide a further test of the experimental calibrations, whereas the consistency of the results for the different charge and $\left|\eta_{\ell}\right|$ categories tests the $W$-boson production model. Four examples together with the overall combination are shown in table 1 listing also the statistical and systematic uncertainties of the measurements. It is interesting to compare the uncertainty difference between the two flavour channels or between the two kinematic variables, and to see the uncertainty improvement for the overall combination. The combination yields $m_{W}=80370 \pm 7$ (stat) \pm 11 (exp. syst) $\pm 14(\bmod$. syst) $[19($ tot $)] \mathrm{MeV}$ and is compatible with the current world average of $m_{W}=80385 \pm 15 \mathrm{MeV}$ [7] and other measurements [8] as shown in figure $3 \mathrm{c}$. The ATLAS measurement has similar precision as the currently leading measurements from $\mathrm{CDF}$ and D0 and has already a significant impact on the world average and the precision of the indirect determination of $m_{W}[9]$. 

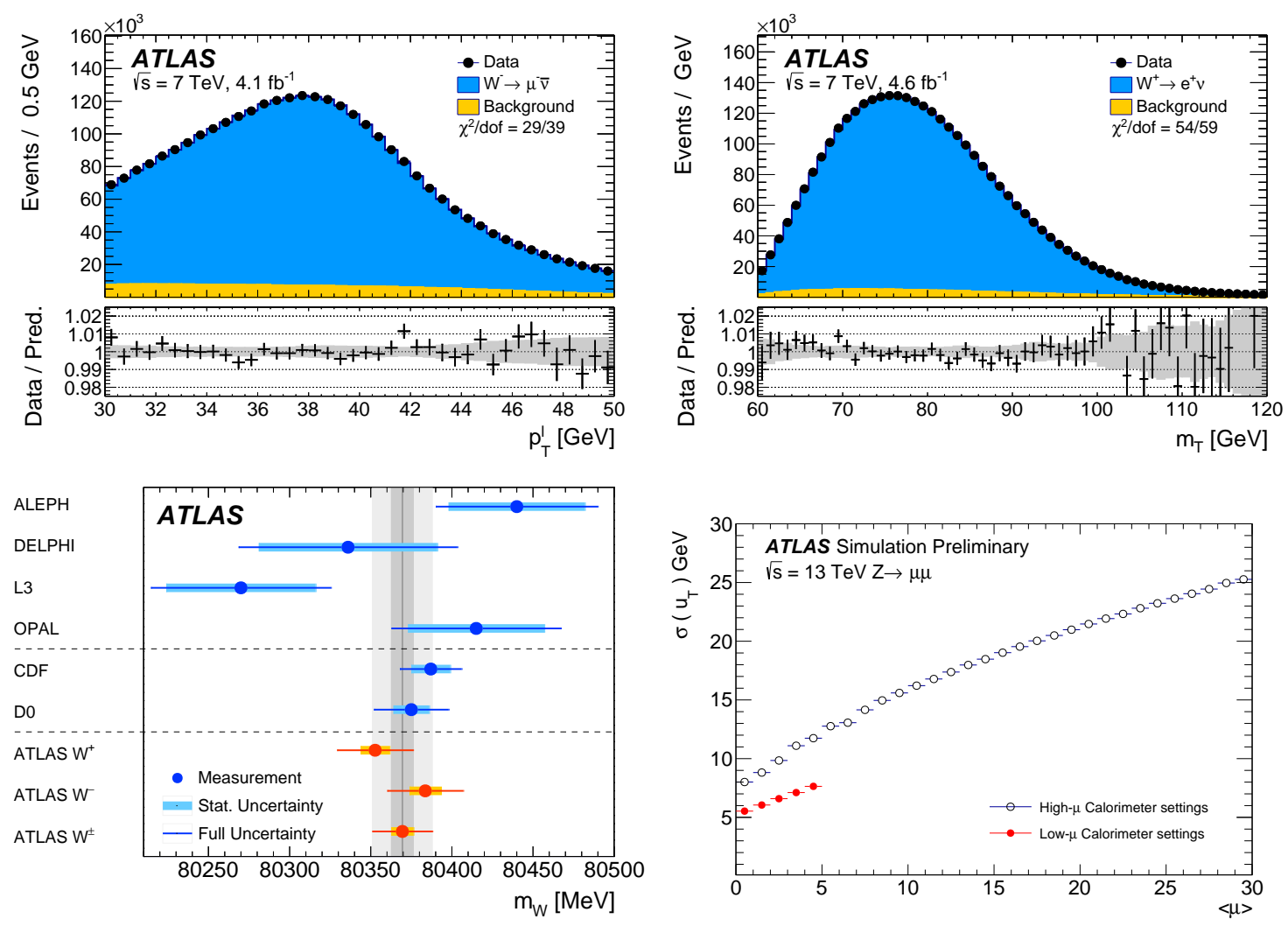

Figure 3: Top-left: $p_{\mathrm{T}}$ distribution for $W^{-}$events in the muon channel. Top-right: $m_{\mathrm{T}}$ distribution for $W^{+}$ events in the electron channel. The data are compared to the simulation including signal and background contributions. Detector calibration and physics-modelling corrections are applied to the simulated events. For both distributions, $m_{W}$ is set according to the overall measurement results. The lower panels show the data-to prediction ratios. The error bars show the statistical uncertainty, and the band shows the systematic uncertainty of the prediction. The $\chi^{2}$ values displayed in each figure account for all sources of uncertainty and include the effects of bin-to-bin correlation induced by the systematic uncertainties. Bottom-left: measured $m_{W}$ compared to other published results. The vertical bands show the statistical and total uncertainties of the ATLAS measurement, and the horizontal bands and lines show the statistical and total uncertainties of the other published results. Measured $m_{W}$ for positively and negatively charged $W$ bosons are also shown. Bottom-right: Recoil resolution as a function of pileup $\langle\mu\rangle$ for simulated $Z \rightarrow \mu \mu$ events with two different calorimeter settings. The plots are taken from $[5,10]$.

\section{Summary and perspectives}

Electroweak precision measurements at the LHC are very challenging. Yet the three examples presented in this talk show that the precision measurements can be achieved with good and competitive precision. The tau polarisation measurement is sensitive to the weak mixing angle and the technique may be used to distinguish beyond SM signals from SM background events. The triple-differential cross section and the forward-background asymmetry of the Drell-Yan events are sensitive to the weak mixing angle and the parton distribution functions and the latter is important for improving the precision of the $W$-boson mass measurement. The $W$-boson mass has been measured with best precision from a single experiment but it needs improvement to match the precision 


\begin{tabular}{l|c|rrrrrrrrr|c}
\hline $\begin{array}{l}\text { Combined } \\
\text { categories }\end{array}$ & $\begin{array}{c}\text { Value } \\
{[\mathrm{MeV}]}\end{array}$ & $\begin{array}{r}\text { Stat. } \\
\text { Unc. }\end{array}$ & $\begin{array}{r}\text { Muon } \\
\text { Unc. }\end{array}$ & $\begin{array}{r}\text { Elec. } \\
\text { Unc. }\end{array}$ & $\begin{array}{r}\text { Recoil } \\
\text { Unc. }\end{array}$ & $\begin{array}{r}\text { Bckg. } \\
\text { Unc. }\end{array}$ & $\begin{array}{l}\text { QCD } \\
\text { Unc. }\end{array}$ & $\begin{array}{r}\text { EW } \\
\text { Unc. }\end{array}$ & $\begin{array}{r}\text { PDF } \\
\text { Unc. }\end{array}$ & $\begin{array}{c}\text { Total } \\
\text { Unc. }\end{array}$ & $\begin{array}{c}\chi^{2} / \text { dof } \\
\text { of comb. }\end{array}$ \\
\hline$p_{\mathrm{T}}^{\ell}, W^{ \pm}, e$ & 80347.2 & 9.9 & 0.0 & 14.8 & 2.6 & 5.7 & 8.2 & 5.3 & 8.9 & 23.1 & $4 / 5$ \\
$m_{\mathrm{T}}, W^{ \pm}, e$ & 80364.6 & 13.5 & 0.0 & 14.4 & 13.2 & 12.8 & 9.5 & 3.4 & 10.2 & 30.8 & $8 / 5$ \\
$p_{\mathrm{T}}^{\ell}, W^{ \pm}, \mu$ & 80382.3 & 10.1 & 10.7 & 0.0 & 2.5 & 3.9 & 8.4 & 6.0 & 10.7 & 21.4 & $7 / 7$ \\
$m_{\mathrm{T}}, W^{ \pm}, \mu$ & 80381.5 & 13.0 & 11.6 & 0.0 & 13.0 & 6.0 & 9.6 & 3.4 & 11.2 & 27.2 & $3 / 7$ \\
\hline$m_{\mathrm{T}}-p_{\mathrm{T}}^{\ell}, W^{ \pm}, e-\mu$ & 80369.5 & 6.8 & 6.6 & 6.4 & 2.9 & 4.5 & 8.3 & 5.5 & 9.2 & 18.5 & $29 / 27$ \\
\hline
\end{tabular}

Table 1: Results of the $m_{W}$ measurements for five selected combinations of categories [5]. The table shows the statistical uncertainties, together with all experimental uncertainties, divided into muon-, electron-, recoil- and background-related uncertainties, and all modelling uncertainties, separately for QCD modelling including scale variations, parton shower and angular coefficients, electroweak corrections, and PDFs. All uncertainties are given in $\mathrm{MeV}$.

of the indirect determinations. There are good perspectives to improve these electroweak measurements with large statistical and dedicated data samples at the LHC. For instance, the low pileup data samples taken recently by ATLAS would allow us to understand better the modelling of low transverse momentum distributions of the $Z$ and $W$ bosons and improve the detector resolution of the hadronic recoil (figure 3d) [10].

\section{References}

[1] M. Baak et al. (Gfitter group), Eur. Phys. J. C 74 (2014) 3046 [arXiv:1407.3792].

[2] ATLAS Collaboration, JINST 3 (2008) S08003; ATLAS-TDR-19, 2010, https://cds.cern.ch/record/1291633, ATLAS-TDR-19-ADD-1, 2012, https://cds.cern.ch/record/1451888.

[3] ATLAS Collaboration, Eur. Phys. J. C 78 (2018) 163 [arXiv:1709.07703].

[4] ATLAS Collaboration, JHEP 12 (2017) 059 [arXiv:1710.05167].

[5] ATLAS Collaboration, Eur. Phys. J. C 78 (2018) 110 [arXiv:1701.07240].

[6] A. Bodek et al., Eur. Phys. J. C 76 (2016) 115 [arXiv:1507.02470].

[7] C. Patrignano et al. (Particle Data Group), Chin. Phys. C 40 (2016) 100001.

[8] CDF Collaboration, T. Aaltonen et al., Phys. Rev. Lett. 108 (2012) 151803; D0 Collaboration, V.M. Abazov et al., Phys. Rev. Lett. 108 (2012) 151804; ALEPH Collaboration, S. Schael et al., Eur. Phys. J. C 47 (2006) 309; DELPHI Collaboration, J. Abdallah et al., Eur. Phys. J. C 55 (2008) 1; L3 Collaboration, P. Achard et al., Eur. Phys. J. C 45 (2006) 569; OPAL Collaboration, G. Abbiendi et al., Eur. Phys. J. C 45 (2006) 307.

[9] J. Haller et al. (Gfitter group), arXiv:1803.01853 [hep-ph].

[10] ATLAS Collaboration, ATL-PHYS-PUB-2017-021, http://cdsweb.cern.ch/record/2298152. 